\title{
Actividad minera y suelos contaminados en el sector de km 100 La Pampa region
}

\author{
Madre de Dios
}

\author{
Mining activity and contaminated soils in the sector kilometer 100 La Pampa \\ region Madre de Dios
}

${ }^{1}$ Lastenia Cutipa Chávez, ${ }^{2}$ Jaime Cuse Quispe

\section{Resumen}

La investigación fue realizada en Madre de Dios tomando como muestra el suelo erosionado y como testigo el suelo no erosionado. El Objetivo de la investigación fue: Determinar la humedad, textura, densidad, pH del suelo en estudio, Determinar el intercambio catiónico, contenido de carbono, contenido de materia orgánica, así como las aproximaciones de Nitrógeno, fosforo y potasio de los suelos del sector, Evaluar la incidencia en los suelos con respecto a los Componentes Físicos y químicos utilizados en la minería. Para el Procedimiento se tomó la muestra al azar cuatro puntos cercanos a una profundidad de $20 \mathrm{~cm}$ haciendo el cuarteo correspondiente, eso para las cinco muestras de los cuales uno es un testigo (muestra $\mathrm{N}^{\circ} 05$ ) obteniendo los siguientes Resultados: Se observó que la humedad de la muestra patrón es $27.05 \%$ y la media de las muestras $(01,02,03$ y 04$)$ de suelos erosionados es $12.46 \%$ esto nos hace ver que existe una variación considerable. Por lo tanto, se Concluye que: La humedad de todas sus formas sufre una alteración en las zonas alejadas de masa de agua, observamos que los suelos erosionados no pueden retener el agua que necesitaría las plantas, La textura de los suelos donde se realizó la actividad minera ha perdido considerable cantidad de limo y arcilla, La materia orgánica que de por si es poca es lamentable, la acidez de los suelos se ve incrementada ligeramente por la actividad minera.

Palabras clave: suelo, muestras, acidez, actividad minera, componentes físicos y químicos.

\section{Summary}

The research was carried out in Madre de Dios taking the eroded soil as a sample and the non-eroded soil as a control. The objective of the investigation was: To determine the humidity, texture, density, $\mathrm{pH}$ of the soil under study, Determine the cation exchange, carbon content, organic matter content, as well as the approximations of Nitrogen, phosphorus and potassium of the soils of the sector, Evaluate the incidence in soils concerning the Physical and Chemical Components used in mining. For the Procedure, the sample was taken at random four points close to a depth of $20 \mathrm{~cm}$, making the corresponding quartering, that for the five samples of which one is a control (sample No. 05) obtaining the following Results: It was observed that The humidity of the standard sample is $27.05 \%$ and the average of the samples $(01,02,03$ and 04$)$ of eroded soils is $12.46 \%$, which makes us see that there is considerable variation. Therefore, it is concluded that: The humidity of all its forms undergoes an alteration in the areas far from the water body, we observe that the eroded soils cannot retain the water that the plants would need, The texture of the soils where the Mining activity has lost a considerable amount of silt and clay. The organic matter, which in itself is little, is unfortunate, the acidity of the soils is slightly increased by mining activity.

Keywords: soil, samples, acidity, mining activity, physical and chemical components.

\footnotetext{
${ }^{1}$ Dra. En Ciencias de la Educación, químico. Departamento Académico de Enfermería. Universidad Nacional Amazónica de Madre de Dios, Perú. Email: lcutipa@unamad.edu.pe / lasteniacch@gmail.com

${ }^{2}$ Dr. En Ciencias de la educación, Lic. En educación, Especialidad Geografía, -Miembro Activo de la Sociedad Geográfica de Lima. Docente de la Universidad Nacional de San Martin - Tarapoto. Email: jcuseq@unsm.edu.pe /

jaimebarrionuevo771@hotmail.com
} 


\section{INTRODUCCIÓN}

Actualmente la región de Madre de Dios catalogado como capital de la Biodiversidad según ley 26311 tiene como actividad económica de mayor desarrollo la minería que debería realizarse en el corredor minero, sin embargo la zona denominada "la Pampa" es una zona de amortiguamiento de la reserva de Tambopata, esta actividad ya no es artesanal, se emplea maquinaria como motobombas, carrancheras hasta dragas que son maquinaria pesada, esta actividad consiste en lavar el suelo donde hay contenido de oro, lo que hace que el suelo es prácticamente degradado por el lavado de todo el material orgánico así como la arcilla y el limo quedando prácticamente montículos de arena y cascajo, a esto se suma la deforestación si en dichos suelos encuentran algún rastro de oro quedando a su paso un desierto pelado. Al observar estos suelos degradados donde la foresta se ha arrasado cambiando el paisaje y el ecosistema de dichos lugares y como no existe programas de reforestación de dichas zonas devastadas por la actividad minera tanto informal como ilegal en la zona la pampa, se realizó el trabajo de investigación de dichos suelos en sus parámetros físicos, químicos y contenido de elementos pesados en la zona del kilómetro 100 cercana a la zona de la pampa. Es necesario mencionar que para entrar a la zona donde se realiza esta actividad uno tiene que pasar desapercibido porque a pesar de que la zona tiene accesos estos son controlados por los mismos Número de muestras (04) en zona de actividad minera; las cuatro muestras se recogieron de suelo erosionado donde se realizó la actividad minera, una de ellas se tomó cerca de una pequeña masa de agua denominada como muestra $\mathrm{N}^{\circ} 03$; y las otras tres muestras son las Muestras $\mathrm{N}^{\circ} 01$, Muestra $\mathrm{N}$ de coordenadas ${ }^{\circ} 02$ mineros ilegales y no dejan ingresar a cualquier persona, no obstante nos ubicamos en zonas que fueron abandonados por la explotación donde se tomó las coordenadas correspondientes con un GPS el mismo que se muestra un croquis del muestreo realizado, se consideró cuatro muestras problema de los cuales tres con similares características a simple observación y el cuarto se tomó alrededor de una zona inundada donde se había realizado dicha actividad minera y una muestra de testigo que se tomó de un área cercana a un bosque que tampoco era prístino sin embargo el suelo de dicho lugar no estaba impactado por la minería.

Estas muestras tomadas luego de ser secadas se procedieron a realizar los análisis de los parámetros físicos como humedad, densidad aparente, densidad real, capilaridad, higroscopicidad, etc. Así como los parámetros químicos como caliza, $\mathrm{pH}$, materia orgánica entre otros y la presencia de metales y otros elementos detectados.

\section{MATERIAL Y MÉTODO}

El lugar de estudio de los suelos producto de la actividad minera aurífera se ubica en el km 100 de la ciudad de Puerto Maldonado de la carretera Puerto Maldonado-Cusco al margen derecho de la carretera cuando se dirige a la ciudad del Cusco, se toma como puntos a los lugares donde se hicieron el muestreo y a continuación se considera como las coordenadas: Entrada a la

\section{zona: punto 853; Población: punto 854}

y Muestra $\mathrm{N}^{\circ} 04$ se tomaron lejos de la masa de agua, dicha masa de agua se observó que fue producto de la actividad minera porque estaba atrapada en medio de suelos erosionados; a continuación se hace referencia como puntos de toma de muestra que se detalla a continuación: 
Tabla 01: puntos tomados con GPS (Ver grafica 01)

\begin{tabular}{|c|c|c|c|c|}
\hline Muestra $N^{\circ} 01$ & Muestra $N^{\circ} 02$ & Muestra $N^{\circ} 03$ & Muestra $N^{\circ} 04$ & Muestra $N^{\circ} 05$ \\
\hline Pto (1) 855 & Pto (1) 859 & Pto (1) 863 & Pto (1) 865 & Pto (1) 869 \\
\hline Pto (1) 856 & Pto (1) 860 & Pto (1) 864 & Pto (1) 866 & Pto (1) 870 \\
\hline Pto (1) 857 & Pto (1) 861 & & Pto (1) 867 & Pto (1) 871 \\
\hline Pto (1) 858 & Pto (1) 862 & & Pto (1) 868 & Pto (1) 872 \\
\hline
\end{tabular}

Muestra de referencia o denominada muestra Patrón también Testigo dicha muestra se extrajo de una zona de bosque natural, se trata de una sola muestra denominada como Muestra $\mathrm{N}^{\circ} 05$.

A continuación se pasa a mostrar un pequeño mapa de donde se obtuvo las muestras para el estudio de los suelos erosionados por la actividad minera comparados con un testigo obtenido de una zona forestada; estos lugares están ubicados en el área que corresponde al $\mathrm{km} 100$ de la carretera de Puerto Maldonado a Cusco que se ubica en la gráfica $\mathrm{N}^{\circ} 01$.

GRÁFICA No01 MAPA DE UBICACIÓN DE MUESTRAS DE SUELO KM 100

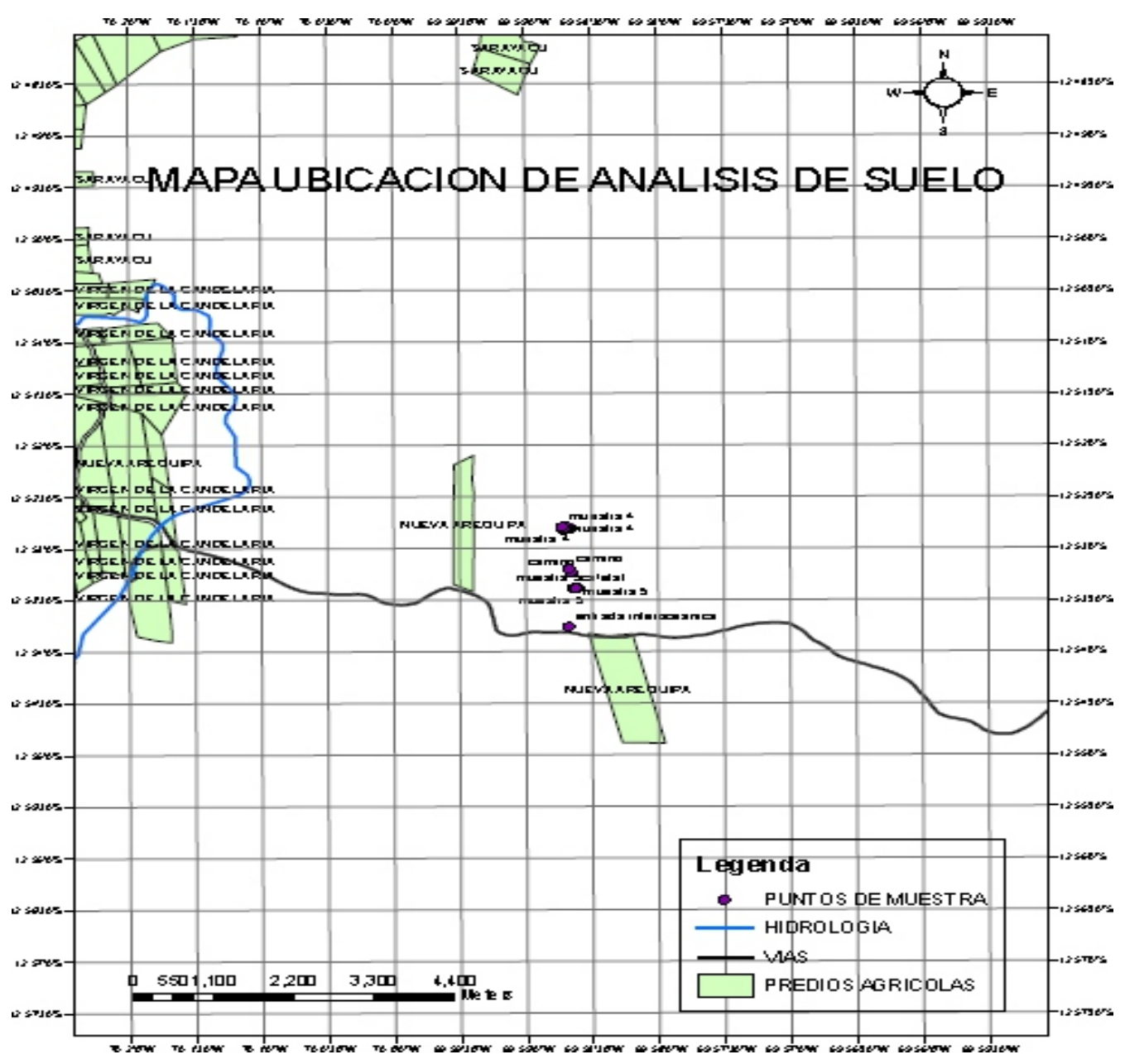




\section{Métodos}

Para el presente estudio se tomó la muestra al azar de cuatro puntos cercanos a una profundidad de $20 \mathrm{~cm}$ los mismos que se mezclan y se hace el cuarteo correspondiente eso para las cinco muestras de los cuales uno es un testigo (muestra $\mathrm{N}^{\circ} 05$ ) y los otros erosionados tres de ellos similares (muestra $\mathrm{N}^{\circ} 01$, Muestra $\mathrm{N}^{\circ} 02$, Muestra $\mathrm{N}^{\circ} 04$ ) y el tercero (Muestra $\mathrm{N}^{\circ} 03$ ) cerca de una masa de agua artificial producto de la actividad antrópica.

\section{Determinación de humedad de un suelo}

La humedad del suelo se determinó por el método clásico gravimétrico. Determinando la humedad por evaporación del agua contenida, en una estufa a $105^{\circ} \mathrm{C}$ expresando los resultados en forma de porcentajes, respecto al peso del suelo desecado.

\section{Cálculos}

Datos:

$\mathrm{P}=$ Peso de muestra antes de

secar.

$\mathrm{P}=$ peso de muestra luego de

secar.

$$
\% \text { Humedad }=\frac{P-p}{P} x 100 \%
$$

\section{DETERMINACIÓN DE LA TEXTURA DE LOS SUELOS}

FUNDAMENTO.- En el análisis mecánico de suelos, el primer objetivo fue la determinación de la textura de los mismos, es decir la distribución por tamaño de las partículas aisladas que forman. Para lo cual se utilizó el método del densímetro del tipo Bouyoucos.

\section{Cálculos}

\section{Datos:}

$\mathrm{C}=$ Medida del densímetro, a los 40 segundos. 24

$\mathrm{C}^{\prime}=$ Medida del densímetro, a las 2 horas. 16.5

$\mathrm{T}=$ Temperatura en 40 seg. $16^{\circ} \mathrm{C}$

$\mathrm{T}^{\prime}=$ Temperatura en 2 horas. $16.5^{\circ} \mathrm{C}$

$$
\begin{gathered}
\%\left(\text { limo y arcilla) es igual } a: \frac{C+(T-20) \times 0.36}{50} \times 100 \%\right. \\
\% \text { arcilla } Y: \frac{C^{\prime}+\left(T^{\prime}-20\right) \times 0.36}{50} \times 100 \%
\end{gathered}
$$

$\mathrm{X}-\mathrm{Y} \quad=$ Porcentaje de limo. $100-\mathrm{X}=$ Porcentaje de arena.

$0.36=$ Factor de corrección por grado de diferencia de temperatura. 
Se supone que el constante del densímetro es de $20^{\mathrm{a}} \mathrm{C}$.

Con los respectivos porcentajes de las fracciones arena, limo y arcilla empleando el triángulo de texturas determinar la clase textural del suelo, la misma que estará dada por el área en el cual se interceptan las 3 líneas.

\section{DETERMINACIÓN DE FÓSFORO PROCEDIMIENTO:}

Se Pesó 5 gr de muestra y añadió $25 \mathrm{ml}$ de solución extractora, luego se filtró y realizo la curva de calibración, utilizando $1 \mathrm{ml}$ del filtrado agregando $2 \mathrm{ml}$ de ácido ascórbico y $2 \mathrm{ml}$ de molibdato y se completó a $25 \mathrm{ml}$ a $45^{\circ} \mathrm{C}$ en baño maría por 10 a 30 minutos.

Los resultados se determinan como $\mathrm{P}_{2} \mathrm{O}_{5}$ sea considerando la absorbancia o tranmitancia

Tabla 02 Calibración Para Determinar Fosforo

\begin{tabular}{|c|c|c|c|c|}
\hline $\begin{array}{c}\text { Nro de } \\
\text { tubo }\end{array}$ & $\begin{array}{c}\text { Patron P } \\
(\mathbf{m l})\end{array}$ & $\begin{array}{c}\text { Muestra } \\
\text { problema } \\
(\mathbf{m l})\end{array}$ & $\begin{array}{c}\text { Molibdato de } \\
\text { amonio (m) }\end{array}$ & $\begin{array}{c}\text { Ácido } \\
\text { Ascórbico }\end{array}$ \\
\hline Blanco & - & - & 2.5 & 2.5 \\
\hline 1 & 0.01 & - & 2.5 & 2.5 \\
\hline 2 & 0.03 & - & 2.5 & 2.5 \\
\hline 3 & 0.05 & - & 2.5 & 2.5 \\
\hline 4 & 0.10 & - & 2.5 & 2.5 \\
\hline 5 & 0.15 & - & 2.5 & 2.5 \\
\hline 6 & - & 1.0 & 2.5 & \\
\hline
\end{tabular}

\section{DETERMINACIÓN DE POTASIO PROCEDIMIENTO:}

A 10 gramos de muestra se agregó $20 \mathrm{ml}$ de solución extractora, se agito por 10 minutos, se filtró, evaporo $5 \mathrm{ml}$ hasta $1 \mathrm{ml}$; ; se midió un $\mathrm{ml}$ del filtrado y se llevó a baño de hielo, luego se añadió 4 gotas de cobaltinitrito de sodio más cinco $\mathrm{ml}$ de alcohol isopropílico (también se puede añadir etanol al 70\%) se dejó por espacio de 10 minutos, previamente agitado realizando la lectura a $660 \mathrm{~nm}$. La curva de calibración se efectúo con una solución de 1 $\mathrm{mg} / \mathrm{ml}$.

\section{ANÁLISIS CUALITATIVO DE CONTENIDO DE METALES}

Se hizo en un equipo de medición espectroscópica y el electrón voltios que tiene los elementos químicos, empleando el equipo InnovX systems serial 512299; las determinaciones se realizaron para cada una de las muestras por una repetición de tres como mínimo, para obtener un promedio por muestra. 


\section{RESULTADOS}

A continuación los valores de humedad obtenidos en el presente trabajo de investigación se detallan en la tabla 03:

Tabla 03: Porcentaje De Humedad

\begin{tabular}{|l|r|r|r|}
\hline MUESTRA & $\begin{array}{r}\text { \% HUMEDAD } \\
\text { (luna de reloj) }\end{array}$ & $\begin{array}{r}\text { \% HUMEDAD } \\
\text { (Crisol) }\end{array}$ & $\begin{array}{r}\text { \% HUMEDAD } \\
\text { PROMEDIO }\end{array}$ \\
\hline Muestra $\mathrm{N}^{\circ} 01$ & 7.55 & 7.38 & $\mathbf{7 . 4 7}$ \\
\hline Muestra $\mathrm{N}^{\circ} 02$ & 6.87 & 7.08 & $\mathbf{6 . 9 8}$ \\
\hline Muestra $\mathrm{N}^{\circ} 03$ & 28.52 & 27.31 & $\mathbf{2 7 . 9 2}$ \\
\hline Muestra $\mathrm{N}^{\circ} 04$ & 7.525 & 7.478 & $\mathbf{7 . 5 0}$ \\
\hline Muestra $\mathrm{N}^{\circ} 05$ & 25.558 & 28.539 & $\mathbf{2 7 . 0 5}$ \\
\hline
\end{tabular}

\section{DETERMINACIÓN DE LA DENSIDAD APARENTE}

Tabla 04: Densidad Aparente

\begin{tabular}{|l|c|c|c|c|}
\hline \multicolumn{1}{|c|}{ MUESTRA } & $\begin{array}{c}\text { Densidad } \\
\text { Aparente }\end{array}$ & $\begin{array}{c}\text { Densidad } \\
\text { Aparente }\end{array}$ & $\begin{array}{c}\text { Densidad } \\
\text { Aparente }\end{array}$ & $\begin{array}{c}\text { Densidad aparente } \\
\text { promedio }\end{array}$ \\
\hline Muestra $\mathrm{N}^{\circ} 01$ & $1.449 \mathrm{~g} / \mathrm{ml}$ & $1.437 \mathrm{~g} / \mathrm{ml}$ & $1.454 \mathrm{~g} / \mathrm{ml}$ & $\mathbf{1 . 4 4 7} \mathbf{g} / \mathbf{m l}$ \\
\hline Muestra $\mathrm{N}^{\circ} 02$ & $1.396 \mathrm{~g} / \mathrm{ml}$ & $1.415 \mathrm{~g} / \mathrm{ml}$ & $1.409 \mathrm{~g} / \mathrm{ml}$ & $\mathbf{1 . 4 0 7} \mathbf{~ g / m l ~}$ \\
\hline Muestra $\mathrm{N}^{\circ} 03$ & $2.004 \mathrm{~g} / \mathrm{ml}$ & $1.748 \mathrm{~g} / \mathrm{ml}$ & $1.791 \mathrm{~g} / \mathrm{ml}$ & $\mathbf{1 . 8 4 8} \mathbf{~ g / m l}$ \\
\hline Muestra $\mathrm{N}^{\circ} 04$ & $1.338 / \mathrm{ml}$ & $1.341 \mathrm{~g} / \mathrm{ml}$ & $1.358 \mathrm{~g} / \mathrm{ml}$ & $\mathbf{1 . 3 4 6} \mathbf{~ g / m l}$ \\
\hline Muestra $\mathrm{N}^{\circ} 05$ & $1.119 \mathrm{~g} / \mathrm{ml}$ & $1.143 \mathrm{~g} / \mathrm{ml}$ & $1.133 \mathrm{~g} / \mathrm{ml}$ & $\mathbf{1 . 1 3 2} \mathbf{~ g / m l}$ \\
\hline
\end{tabular}

\section{OBSERVACIONES}

La muestra patrón $\left(\mathrm{N}^{\circ} 05\right)$ tiene menor densidad aparente es razonable dicho valor debido a que en su textura la cantidad de arena, limo, arcilla son los que corresponden al tipo de suelo en bosque primario.

El numero 03 presenta un valor elevado por la presencia de mayor cantidad de agua (humedad) y arena lo que se ve claramente su valor elevado respecto a la muestra $\mathrm{N}^{\circ} 01 ; 02$; 03 


\section{CÁLCULO DE LA POROSIDAD DE UN SUELO}

Tabla 05 Porcentaje De Porosidad

\begin{tabular}{|c|c|c|c|}
\hline MUESTRA & $\begin{array}{c}\text { DENSIDAD } \\
\text { APARENTE } \\
\text { g/ml }\end{array}$ & $\begin{array}{c}\text { DENSIDAD } \\
\text { REAL g/ml }\end{array}$ & \% POROSIDAD \\
\hline $\mathrm{N}^{\mathrm{a} 01}$ & 1,447 & 2,2971 & 37,006 \\
\hline $\mathrm{N}^{\mathrm{a}} 02$ & 1,407 & 2,2543 & 37,587 \\
\hline $\mathrm{N}^{\mathrm{a}} 03$ & 1,848 & 2,0328 & 9,093 \\
\hline $\mathrm{N}^{\mathrm{a}} 04$ & 1,346 & 2,3413 & 42,510 \\
\hline $\mathrm{N}^{\mathrm{a}} 05$ & 1,132 & 2,0207 & 43,981 \\
\hline
\end{tabular}

La variación en la porosidad entre la muestra patrón $\left(\mathrm{N}^{\circ} 05\right)$ y la muestra $\mathrm{N}^{\circ} 03$ es mayor debido a que la muestra $\mathrm{N}^{\circ} 03$ fue tomada cerca de una masa de agua y por lo tanto es muy húmeda, sin embargo entre la muestra patrón $\left(\mathrm{N}^{\circ} 05\right)$ y los suelos impactados por la actividad minera $\left(\mathrm{N}^{\circ} 01,02,04\right)$ la variación que existe es razonable el patrón es mayor y eso indica que existe cambios notorios en este parámetro.

\section{DETERMINACIÓN DE HUMEDAD EN EL SUELO - MÉTODO DE EMMERT}

Es la medición de la elevación de la temperatura que se produce al añadir $\mathrm{H}_{2} \mathrm{SO}_{4}$ (c) con el agua que contiene el suelo. Construcción de la Curva de Emmert. Considerando las variables de \% de humedad y temperatura en ${ }^{\circ} \mathrm{C}$

Tabla 06 Consolidado Humedad Método Emmert

\begin{tabular}{|l|c|c|c|c|c|}
\hline & Muestra No01 & $\begin{array}{c}\text { Muestra } \\
\mathbf{N}^{\circ} \mathbf{0 2}\end{array}$ & $\begin{array}{c}\text { Muestra } \\
\mathbf{N}^{\circ} \mathbf{0 3}\end{array}$ & $\begin{array}{c}\text { Muestra } \\
\mathbf{N}^{\circ} \mathbf{0 4}\end{array}$ & Muestra $\mathbf{N}^{\circ} \mathbf{0 5}$ \\
\hline \% HUMEDAD & 4.1956 & 5.2783 & 8.6029 & 5.0302 & 8.6090 \\
\hline
\end{tabular}

Se puede observar que la muestra $\mathrm{N}^{\circ} 03$ recogida de una zona cercana a una masa de agua la humedad es semejante a la humedad de la muestra patrón o testigo (Muestra $\mathrm{N}^{\circ} 05$ )

\section{DETERMINACIÓN DE LA TEXTURA DE LOS SUELOS}

Tabla 07 Datos Obtenidos De Textura

\begin{tabular}{|c|c|c|c|c|}
\hline $\boldsymbol{N}^{\circ}$ MUESTRA & \% ARCILLA & \% LIMO & \% ARENA & TEXTURA \\
\hline 1 & 7.58 & 0.67 & 91.6 & Arenosa \\
\hline 2 & 16.12 & 2.16 & 83.88 & Arenosa-Franca \\
\hline 3 & 18.72 & 2.4 & 78.88 & Franco - arenosa \\
\hline 4 & 16.2 & 0.24 & 83.52 & Arenosa - franca \\
\hline 5 & 18.12 & 32 & 50.12 & Franca \\
\hline
\end{tabular}




\section{DETERMINACIÓN DEL CONTENIDO EN CARBONO \\ Y MATERIA ORGÁNICA DE LOS SUELOS \\ (MÉTODO DE WALKLEY Y BLACK)}

Tabla 08 Datos De Materia Orgánica

\begin{tabular}{|c|c|c|}
\hline $\mathbf{N}^{\circ}$ & $\%$ M.O & \% M.O PROMEDIO \\
\hline \multirow{3}{*}{1} & 1.62 & \multirow{3}{*}{1.50} \\
\hline & 1.51 & \\
\hline & 1.37 & \\
\hline \multirow{3}{*}{2} & 1.77 & \multirow{3}{*}{1.41} \\
\hline & 1.25 & \\
\hline & 1.22 & \\
\hline \multirow{3}{*}{3} & 2.42 & \multirow{3}{*}{1.94} \\
\hline & 1.94 & \\
\hline & 1.47 & \\
\hline \multirow{3}{*}{4} & 2.28 & \multirow{3}{*}{1.75} \\
\hline & 1.50 & \\
\hline & 1.47 & \\
\hline \multirow{3}{*}{5} & 3.47 & \multirow{3}{*}{3.39} \\
\hline & 3.36 & \\
\hline & 3.35 & \\
\hline
\end{tabular}

\section{RESULTADOS DEL CONTENIDO DE N P y K}

Tabla 09 Determinación De N P K

\begin{tabular}{|c|c|c|c|}
\hline $\mathrm{N}^{\circ}$ MUESTRA & $\begin{array}{l}\text { NITROGENO } \\
\%\end{array}$ & $\begin{array}{l}\text { FOSFORO } \\
\text { ppm } \mathrm{P}_{2} \mathrm{O}_{5}\end{array}$ & $\begin{array}{l}\text { POTASIO } \\
\text { ppm K } \mathrm{K}_{2} \mathrm{O}\end{array}$ \\
\hline 01 & 0.020 & 0.31 & 19.20 \\
\hline 02 & 0.011 & 0.17 & 12.60 \\
\hline 03 & 0.025 & 0.39 & 3.80 \\
\hline 04 & 0.022 & 0.34 & 6.20 \\
\hline 05 & 0.036 & 0.56 & 59.40 \\
\hline
\end{tabular}

\section{DISCUSIÓN}

Al realizar la comparación de la muestra patrón (muestra $\mathrm{N}^{\circ} 05$ ) y las muestras $\mathrm{N}^{\circ} 01,02$ ,04 y 03 (extraída de un lugar cercano a un banco de agua), por el método T student de comparación de pares se tiene los siguientes resultados en el cuadro $\mathrm{N}^{\circ} 005$

Se observa que la humedad de la muestra patrón es $27.05 \%$ y la media de las muestras $(01$, $02,03$ y 04$)$ de suelos erosionados es $12.46 \%$; esto nos hace ver que existe una variación considerable.

La muestra $\mathrm{N}^{\circ} 03$ de entre la $\mathrm{N}^{\circ} 01, \mathrm{~N}^{\circ} 02$ y $\mathrm{N}^{\circ} 04$ se extrajo de un lugar cercano a agua estancada por lo que es razonable dicha humedad de $27.92 \%$ 
Sin embargo la muestra $\mathrm{N}^{\circ} 05$ es el suelo de comparación porque el lugar donde se extrajo es un suelo de bosque primario con árboles forestales.

Nos hace ver que la erosión producto de la acción antrópica y la textura que presentan se ve afectado en la humedad disponible que deberían tener si dicha actividad no hubiese cambiado la textura de los suelos.

La densidad del suelo que se analiza como patrón es $2.0207 \mathrm{~g} / \mathrm{ml}$ casi similar a la muestra $\mathrm{N}^{\circ} 03$ debido a que este último tiene mayor contenido de agua; sin embargo las muestras erosionadas $\left(\mathrm{N}^{\circ} 01,02\right.$ y 04$)$ existe una variación significativa esto debido a la mayor presencia de arena.

La variación en la porosidad entre la muestra patrón $\left(\mathrm{N}^{\circ} 05\right)$ y la muestra $\mathrm{N}^{\circ} 03$ es mayor debido a que la muestra $\mathrm{N}^{\circ} 03$ fue tomada cerca de una masa de agua y por lo tanto es muy húmeda, sin embargo entre la muestra patrón $\left(\mathrm{N}^{\circ} 05\right)$ y los suelos impactados por la actividad minera $\left(\mathrm{N}^{\circ} 01,02,04\right)$ la variación que existe es razonable el patrón es mayor y eso indica que existe cambios notorios en este parámetro.

Se puede observar que la muestra $\mathrm{N}^{\circ} 03$ recogida de una zona cercana a una masa de agua, la humedad es semejante a la humedad de la muestra patrón o testigo (Muestra $\mathrm{N}^{\circ} 05$ ).

Debido a que los suelos de las muestra $\mathrm{N}^{\circ} 01,02,04$ tienen la misma textura y similar humedad es razonable que su capilaridad sea semejante y difiere de la muestra $\mathrm{N}^{\circ} 03$ a pesar de tener la misma textura; la muestra $\mathrm{N}^{\circ} 05$ es el patrón y la capilaridad es semejante a la muestra $\mathrm{N}^{\circ} 03$ porque esta tiene semejante humedad pero difieren en su textura.

\section{CONCLUSIONES}

La humedad de todas sus formas sufre una alteración en las zonas alejadas de masa de agua, dado que las plantas obtienen del agua disponible que existe en el suelo, observamos que los suelos erosionados no pueden retener el agua que necesitaría las plantas.

La textura de los suelos donde se realizó la actividad minera ha perdido considerable cantidad de limo y arcilla.

La materia orgánica que de por si es poca es lamentable la perdida por la actividad de la minería que se observa en los resultados.

La acidez de los suelos se ve incrementada ligeramente por dicha actividad

En cuanto al contenido de metales no se observa una presencia considerable comparando con los límites permisibles por ello se debe realizar de forma más exhaustiva. 


\section{REFERENCIAS BIBLIOGRÁFICAS}

Barea, J. (1999). Importancia de las micorrizas arbusculares en el establecimiento y protección de las plantas en suelos degradados. Phytoma 111:29-56.

Grim, E. R. (1953). Clay Mineralogy.McGraw-Hill, New York.

Hernández A.J. Y Pastor, J. (2008). La restauración en sistemas con suelos degradados: estudio de casos en agro ecosistemas mediterráneos y taludes de carretera. En: Contaminación de Suelos. Tecnologías para su Recuperación. (eds. R. Millán y C. Lobo): Ed. CIEMAT, Madrid.

Instituto Geográfico Agustín Codazzi. (1998). Estudio general de suelos y zonificación de tierras, Departamento de Sucre. Instituto Geográfico Agustín Codazzi, Sincelejo.

Jackson, M. L. (1970). Análisis Químico de Suelos. Omega Barcelona.

Martínez R. C. (2000). Dinámica de la recuperación de zonas degradadas por movimientos de tierra: sucesión vegetal y clasificación de especies según su actividad colonizadora Tesis Doctoral, Universidad de Salamanca.

Miralles M. I. (2006). “Calidad de suelos en ambientes calizos mediterráneos: Parque Natural de Sierra María-Los Vélez” Tesis Doctoral Granada.

Porta, J; López-Acevedo, Marta; Roquero, Carlos (2003). "Edafología para la agricultura y el medio ambiente", Ed. Mundiprensa, $3^{\text {a }}$ edición.

Totola, M.; Chaer, G. (2002). Microrganismos e procesos microbiológicos como indicadores da qualidade dos solos. Rev. Brasil. Cienc. Solo 2:249-259.

Valdivia M. (2005). Trabajo de investigación metales pesados en el suelo. Rev. Soc. Per. Med. Inter. Perú.

Walkley, A. I. A. Black. (1934). Soil Science An examination of the Degthareff method for determining soil organic matter and a proposed modification of the chromic acid titration method.

\section{REVISTAS Y PÁGINAS WEBB}

http://www.monografias.com/trabajos96/fitoextraccion-metales-pesados-suelos-

contaminados/fitoextraccion-metales-pesados-suelos- contaminados2.shtml\#ixzz33LEFieaL

Articulo Cientifico: Relación Entre Erosion Y Perdida De Fertilidad Del Suelo L. do Prado

Wildner y M. da Veiga R.C. Suelo Nutr. Veg. 6 (2) 2006 (1-12)

Articulo Cientifico: Movilizacion De Hg En Suelos Contaminados Por Actividades Mineras

Navarro, A. Mendoza, J. L. y Domenech, L. 